\title{
A Clinical Pathway for Fabrication of Immediate Complete Denture
}

\author{
${ }^{1}$ Ilkin Tuncel, ${ }^{2}$ Gozde Celik
}

\begin{abstract}
Immediate complete dentures have a significant role when patient's all teeth should be extracted. The dentures are fabricated before all the remaining teeth were extracted, and immediately delivered to the patient after the extraction procedure. This article presents a practical fabrication method for complete immediate denture with the use of patient's existing fixed partial denture (FPD). Advantages and disadvantages of this treatment method were emphasized.
\end{abstract}

Keywords: Complete denture, Immediate denture, Prosthodontics.

How to cite this article: Tuncel I, Celik G. A Clinical Pathway for Fabrication of Immediate Complete Denture. Int J Prosthodont Restor Dent 2015;5(2):60-62

\section{Source of support: Nil}

Conflict of interest: None

\section{INTRODUCTION}

Immediate complete denture is a restoration, which is constructed to be immediately inserted to the patient's mouth, following the extraction of all the remaining natural teeth. Two types of immediate dentures are described in the literature: conventional immediate dentures and interim immediate dentures. ${ }^{1}$ Conventional immediate denture is immediately delivered dentures after the extraction of natural teeth. This type prosthesis can be refitted after the healing period for long-term prosthesis. Interim immediate denture is used for a short time, especially for the healing period. At the end of the healing period, the immediate denture may be relined or replaced with a new complete denture. ${ }^{2}$

Immediate dentures have several advantages. After the extraction of the teeth, immediate denture prevents bleeding, protects the extraction site from trauma, and reduces alveolar ridge resorption. As a result of this,

\footnotetext{
${ }^{1}$ Assistant Professor, ${ }^{2}$ Associate Professor

1,2Department of Prosthodontics, School of Dentistry, Bezmialem Vakif University, Istanbul, Turkey
}

Corresponding Author: Ilkin Tuncel, Assistant Professor Department of Prosthodontics, Faculty of Dentistry, Bezmialem Vakif University 34093, Fatih, Istanbul, Turkey, Phone: 902124531700, e-mail: ilkint@hotmail.com smooth and formed ridges can be obtained. ${ }^{3-6}$ Immediate denture facilitates speaking and nutrition during the healing period. ${ }^{3}$

From the esthetical point of view, prostheses cannot be fully assessed before completion, so the treatment outcome is not always predictable. ${ }^{7}$ Patient's facial view, muscular tone, height and centric relation is transferred to the new denture by using the patient's old fixed partial denture (FPD) with the presented technique. This fast and economical technique satisfies patients by reason of using patient's teeth at the original position.

Several techniques have been reported in the literature for the fabrication of immediate complete denture. Gooya et al described the use of a patient's fixed prosthesis for fabricating an interim immediate partial denture in one appointment, where three remaining teeth were maintained to preserve occlusal vertical dimension (OVD) and retentive clasps were used to improve temporary prosthesis retention. The authors concluded that in this procedure the occlusion, OVD, and facial support was maintained during the healing period. $^{1}$

A speedy, economical interim immediate denture using a vacuum forming machine without conventional laboratory procedures was also reported by Joffe. ${ }^{8}$ Khan et $\mathrm{al}^{9}$ fabricated an immediate transitional complete denture in one appointment using self-polymerizing, tooth-colored acrylic, and visible light-cured resins. Gilboa et $\mathrm{al}^{10}$ performed a procedure to fabricate an immediate complete overdenture using several teeth retained an interim provisional FPD until the complete denture was finished. In this procedure the posterior occlusion was maintained during the healing period and the trauma of multiple extractions at one visit was avoided.

The current article describes a procedure that was used to replicate patient's original vertical dimension, phonetic and esthetic of the existing fixed prostheses as a part of an interim immediate complete denture.

\section{CLINICAL REPORT}

A 52-year-old male presented with maxillary full-mouth FPD supported by teeth 16, 13 and 26 (Fig. 1). Clinical and radiographic evaluation revealed generalized severe 


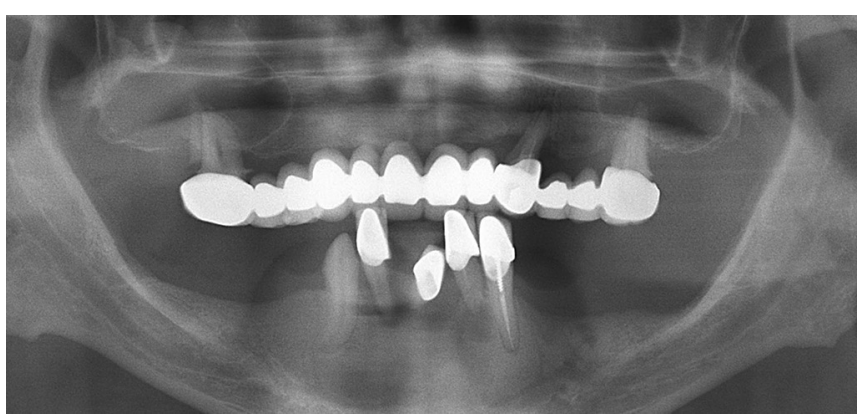

Fig. 1: Initial panoramic radiograph of the patient

chronic periodontitis of the teeth supporting FPD. Periodontal pocket depths of the maxillary teeth were measured between 4 and $8 \mathrm{~mm}$. The extraction of all maxillary teeth was indicated. The patient decided on maxillary and mandibular implant supported FPD among several treatment options. Due to the patient's business and social status, an immediate complete denture by using patient's old FPD was planned. By this way, the same appearance of patient's old prosthesis transferred to the immediate complete denture.

The primary impression including all complete denture borders was made using irreversible hydrocolloid

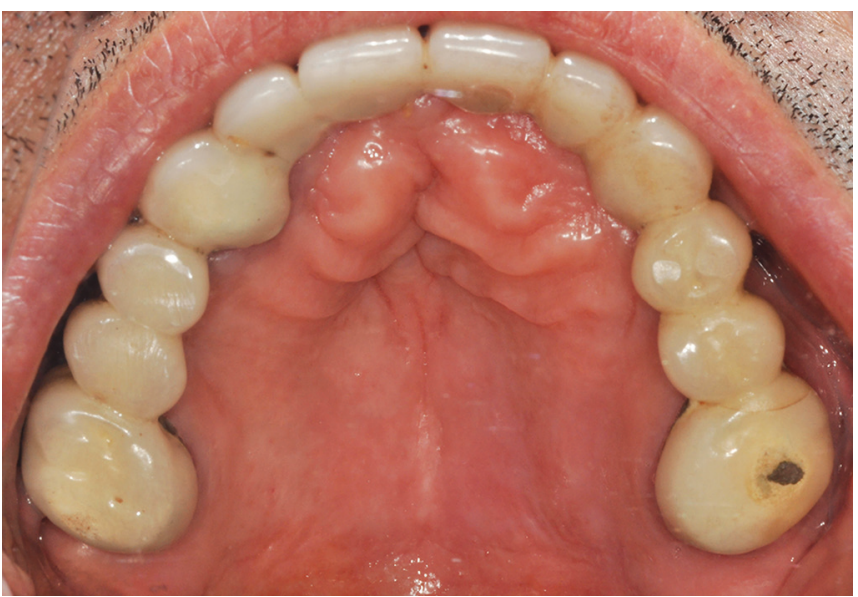

Fig. 2: Intraoral view with existing fixed partial denture

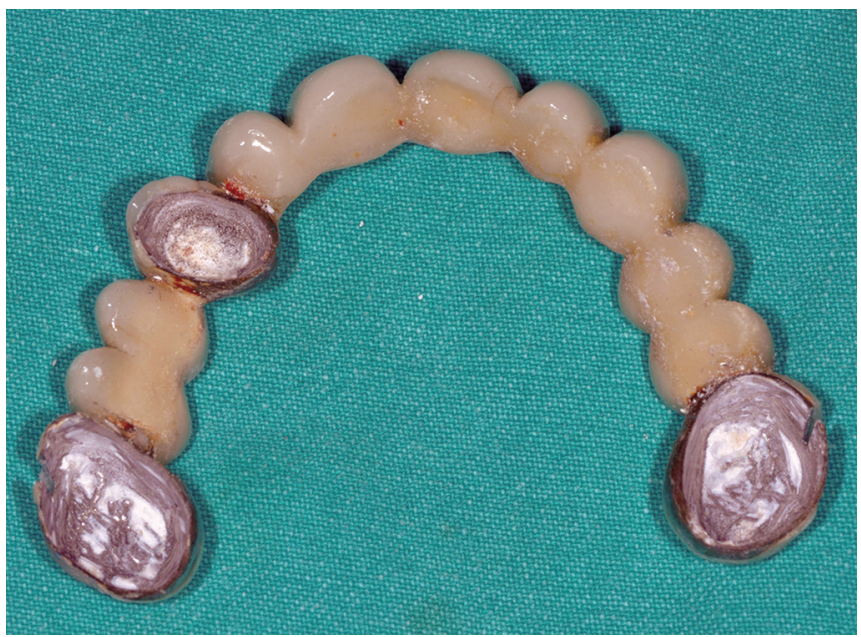

Fig. 4: Removed fixed partial denture
(Alginoplast, Heraeus Kulzer GmbH, Wehrheim, Germany) before maxillary teeth extraction. Maxillary full mouth FPD was removed by cutting the FPD from the most posterior teeths' buccal surface (Figs 2 to 4 ). The space of the extracted teeth of the removed FPD was filled with an autopolymerizing acrylic resin and FPD was placed into the impression (Fig. 5). Stone cast with FPD were obtained using type IV dental stone (Fig. 6). The cast was isolated. (Elite Model, Zhermack, Badia Polesine, Italy)

Autopolymerizing acrylic resin (Meliodent Heraeus Kulzer GmbH, Wehrheim, Germany) was directly applied onto the stone cast and around the FPD on the cast like gingival tissue as a complete denture procedure. Sulcus borders were filled with acrylic resin. After acrylic polymerization, denture removed from the cast, contour and border of the prosthesis were proofed with the suitable thickness. The complete denture was finished and polished in a conventional manner (Fig. 7).

After preparation of the prosthesis, extraction of the teeth was done as atraumatic as possible. The denture was inserted, and post insertion adjustments were made (Fig. 8). Follow-up appointments were scheduled for every 1 month, until the implant surgery.

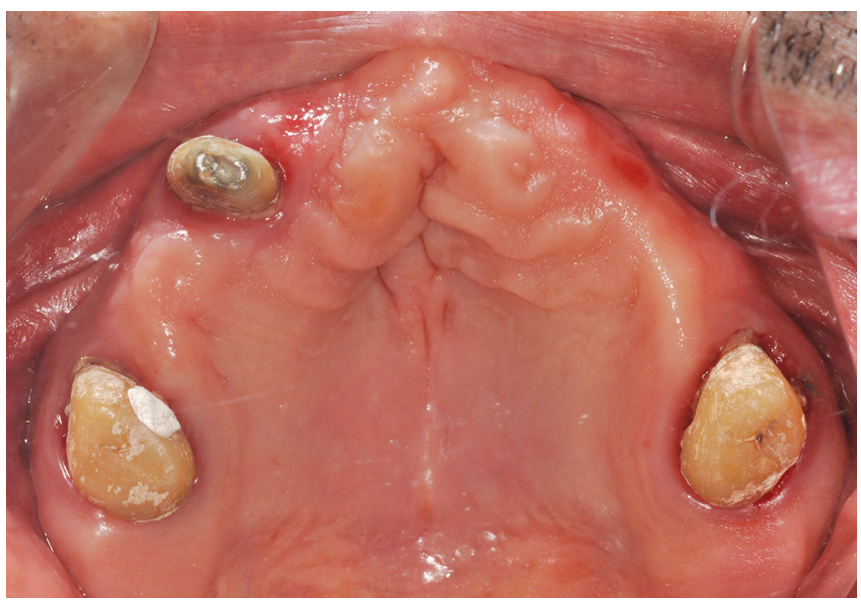

Fig. 3: Intraoral view of supporting teeth

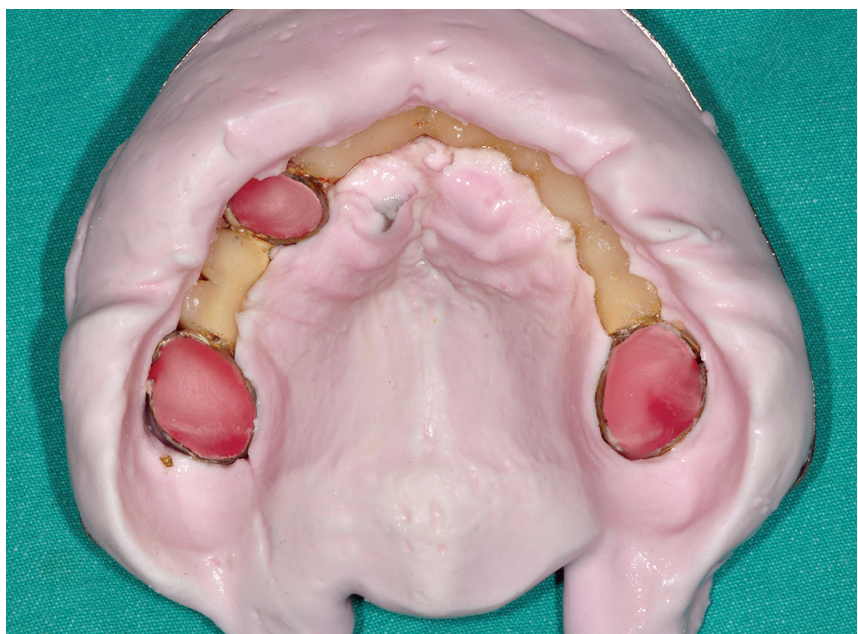

Fig. 5: Internal view of the impression with fixed partial denture 


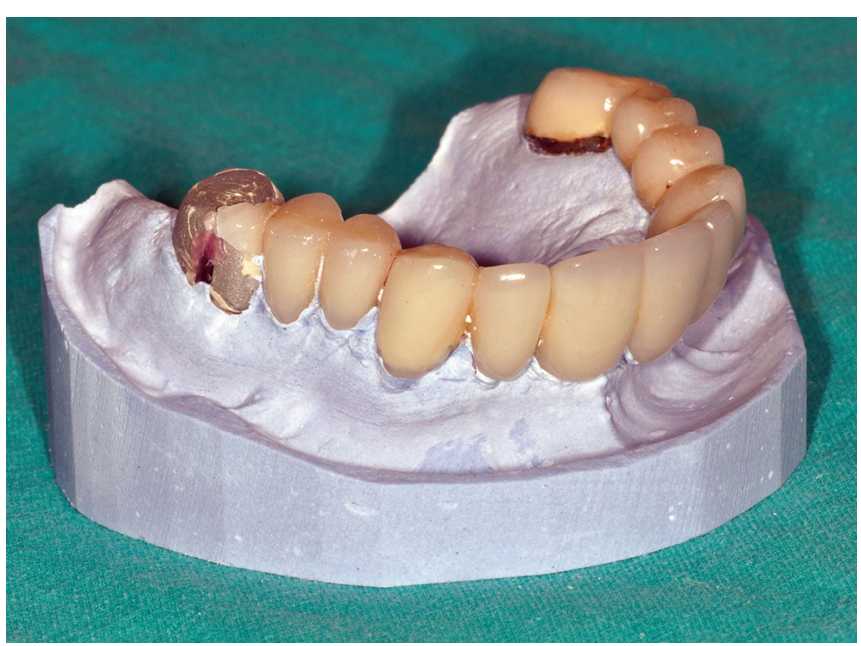

Fig. 6: Prepared stone cast

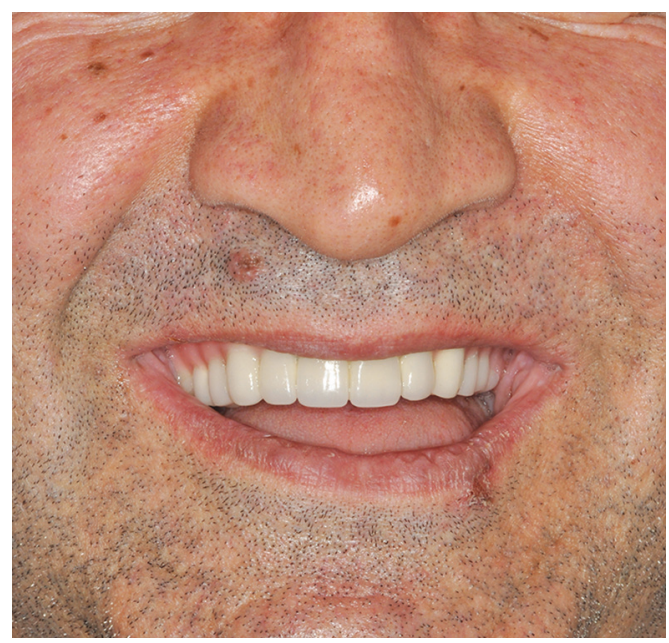

Fig. 8: Completed denture in the mouth

\section{DISCUSSION}

Interim immediate complete dentures provide a reliable treatment option. Presented technique eliminates extra appointments for fabrication. One impression containing the old FPD and the total denture borders was sufficient for making this immediate complete denture.

Patient's original condition was preserved and transferred to the new prosthesis. There was no need to transfer the models to the articulator, determination for centric relation or vertical dimension. Therefore, the clinical and laboratory time was reduced and individual faults at the fabrication of the prosthesis were avoided.

The position of the old FPD was not changed on the immediate denture. This enabled a predictable esthetic outcome of the new denture. Teeth form and position variations caused by technician were not occurred. Patient was not surprised of the new look of the new denture due to use of his old FPD.

The weight of the upper complete denture was the disadvantage of this case. Metal-based FPD framework, which is heavier than acrylic resin made this denture

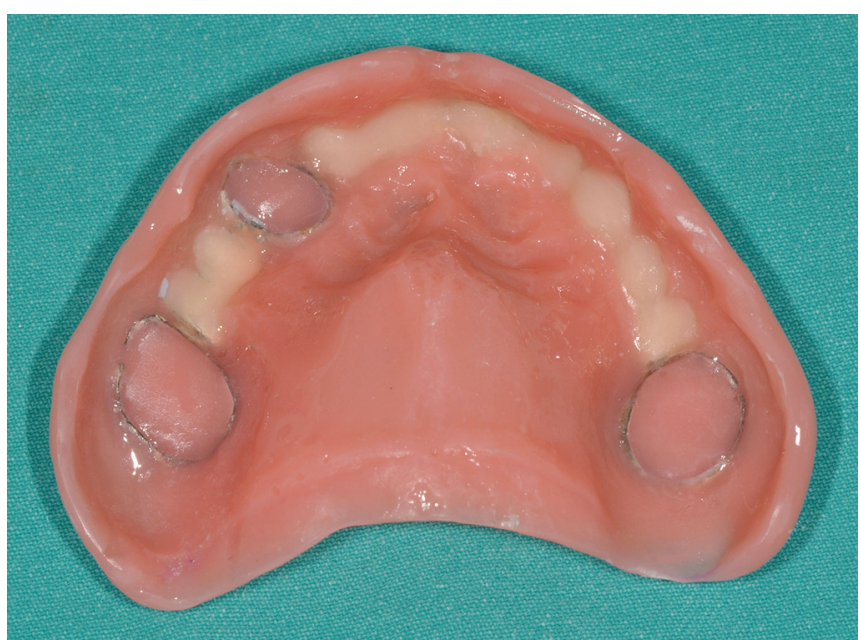

Fig. 7: Completed denture

much heavier than a conventional immediate denture. Due to the reason that, tissue is rapidly changing underneath the immediate denture during healing period, the weight of the denture is very important especially in the maxillary arch. The adaptation of denture and the tissue may be compromised related to the weight of this upper denture.

The technique used in this case report decreased the chair and laboratory time to only one appointment. It was easy and economical. This procedure replicated patient's original vertical dimension, phonetic and esthetic of the existing FPD as a part of the immediate complete denture.

\section{REFERENCES}

1. Gooya A, Ejlali M, Adli AR. Fabricating an interim immediate partial denture in one appointment (modified jiffy denture). A clinical report. J Prosthodont 2013 June;22(4):330-333.

2. Zarb GA, Bolender CL. Prosthodontic treatment for edentulous patients. 12th ed. St. Louis: Mosby; 2004. p. 123-159.

3. Seals RR Jr, Kuebker WA, Stewart KI. Immediate complete dentures. Dent Clin North Am 1996 Jan;40(1):151-167.

4. Loo WD. Ridge preservation with immediate dentures. J Prosthet Dent 1968 Jan;19(1):5-11.

5. Kelly EK, Sievers RF. The influence of immediate dentures on tissue healing and alveolar ridge form. J Prosthet Dent 1959;9(5):738-742.

6. Johnson K. A clinical evaluation of immediate denture procedures. J Prosthet Dent 1966 Sep-Oct;16(5):799-810.

7. Wyatt CCL. Immediate dentures. In MacEntee MI: the complete denture. A clinical pathway. Chicago: Quintessence; 1999. p. 99.

8. Joffe EH. Simplified fabrication of the interim denture using a vacuum forming machine: a clinical report. J Prosthet Dent 1992 Jan;67(6):747-748.

9. Khan X, Haeberle CB. One appointment construction of an immediate transitional complete denture using visible lightcured resin. J Prosthet Dent 1992 Sep;68(3):500-502.

10. Gilboa I, Cardash HS. An alternative approach to the immediate overdenture. J Prosthodont 2009 Jan;18(1):71-75. 